\title{
Infección por Virus de Inmunodeficiencia Humana en Niños y Adolescentes, 30 Años en Chile
}

\author{
Human Immunodeficiency Virus Infection in Children and Adolescents, \\ 30 years in Chile
}

\section{Elba Wu Hupat}

aPediatra Infectóloga, Facultad de Medicina Occidente, U. Chile, Hospital San Juan de Dios Presidenta Comité VIH/SIDA Pediátrico, SOCHIPE

Recibido el 20 de abril de 2017; aceptado el 23 de abril de 2018

\section{Resumen}

El VIH ha sido la gran infección de fines del siglo XX. Esta revisión tiene como objetivos dar a conocer la experiencia del Comité de VIH/SIDA Pediátrico de la Sociedad Chilena de Pediatría desde la detección del primer niño infectado con VIH en Chile, y promover la pesquisa y derivación de los recién nacidos/niños expuestos al VIH y de aquellos infectados con VIH. Se analizan datos actualizados de la literatura nacional y extranjera, presentaciones a Congresos/Talleres efectuadas por el Comité VIH/SIDA Pediátrico, y experiencias de sus integrantes. Se da a conocer la historia del Programa de Atención VIH/SIDA Pediátrico en la pesquisa, diagnóstico, tratamiento y seguimiento desde su creación en 1990. Se presentan los datos de los pacientes detectados desde 1987 al 2008 por el Comité (Cohorte 1) y aquellos obtenidos mediante correo electrónico de los pacientes detectados desde 1987 al 2014 (Cohorte 2). El análisis de estas cohortes permitió concluir que con la aplicación del Programa Preventivo de Transmisión Vertical (PPTV) del VIH ha disminuido la transmisión vertical de este virus desde $>35 \%$ previo a 1995 a $<2 \%$ en la actualidad, y que con el uso de TARV se ha obtenido mejoría clínica, inmunológica y virológica con prolongación de la sobrevida. Finalmente se discuten los logros obtenidos comparando sus resultados con los de la literatura actual, evidenciando que Chile se encuentra a punto de lograr la meta de la eliminación de la transmisión vertical del VIH.

\section{Abstract}

HIV has been the major infection of the late 20th century. This review has the objective of disseminating the experience of the Pediatric HIV/AIDS Committee of the Chilean Pediatric Society since the detection of the first HIV-infected child in Chile and promoting the screening and referral of newborns/children exposed to HIV and those infected with HIV. Updated data from the national and foreign literature, presentations in Congresses/Workshops held by the Pediatric HIV/AIDS Committee,
Palabras clave:

VIH; SIDA;

Transmisión Vertical; Expuestos/Infectados; Antiretrovirales;

Terapia Antiretroviral

Keywords:

HIV;

AIDS;

Vertical Transmission; Exposed/Infected; Antiretroviral; Antiretroviral Therapy 
and experiences of its members are analyzed. It is presented the history of the Pediatric HIV/AIDS Care Program in screening, diagnosis, treatment, and follow-up since its inception in 1990. The data on patients detected from 1987 to 2008 by the Committee (Cohort 1) and those obtained by e-mail from patients detected from 1987 to 2014 (Cohort 2) are presented. The analysis of these cohorts led to the conclusion that with the application of the Preventive Vertical Transmission Program (PPTV) for HIV, the vertical transmission of this virus has decreased from $>35 \%$ prior to 1995 to $<2 \%$ at present, and that clinical, immunological and virological improvement with prolonged survival has been obtained with the use of ART. Finally, we discuss the achievements obtained by comparing the results with those of the current literature, showing that Chile is on the brink of achieving the goal of eradicating the vertical transmission of HIV.

\section{Introducción}

Desde los primeros casos de VIH/SIDA descritos en el mundo (en 1981 en adultos y en 1982 y 1983 en niños, por transfusiones y madre-hijo respectivamente) y hasta el año 2016, habían contraído la infección 76,1 millones de personas y habían fallecido por su causa 35 millones. El 2016, 36,7 millones de personas vivían con $\mathrm{VIH}$, siendo prácticamente la mitad mujeres mayores de 15 años y 2,1 millones de menores de 15 años ${ }^{1,2}$.

\section{VIH/SIDA en Chile}

En Chile los primeros casos de VIH/SIDA en adultos fueron descritos los años 1984 en hombres y en 1985 en mujeres y los primeros casos en niños en 1987 por transfusión y en 1989 por transmisión vertical, madre-hijo ${ }^{3}$.

Según el Ministerio de Salud (MINSAL) cerca de 65.000 personas viven con VIH [41.681 confirmadas por el Instituto de Salud Pública (ISP)] y de ellas 34.301 están en control en la red de salud pública; $62 \%$ diagnosticadas en etapa No SIDA y $38 \%$ en etapa SIDA ${ }^{4,5}$.

Desde 1987 y hasta Junio del 2017, según cifras del ISP, se han pesquisado 414 niños infectados con VIH (388 por trasmisión vertical, 26 por otras causas) ${ }^{6}$.

Según la clasificación de 1994 del Center for Diseases Control and Prevention (CDC) de Estados Unidos, la infección por VIH en niños es la que ocurre en menores de 13 años ${ }^{7}$. En Chile, los pediatras deben controlar a los niños y adolescentes hasta los 18 años [Guías MINSAL 2005 y posteriores ${ }^{8}$ ], incorporando así tanto a infecciones adquiridas por transmisión vertical y por otras causas.

En Chile, la principal vía de transmisión en adultos es sexual (99\%) y en niños, vertical (95\%) 3 . La tendencia a la feminización de la infección por VIH se tradujo en un aumento del número de niños expuestos al VIH/ infectados con $\mathrm{VIH}^{3}$. Pero gracias a la implementación en 1995 de Protocolos de Prevención de la Transmisión Vertical (PPTV) del VIH ${ }^{9-12}$ [incorporado como
Norma por el MINSAL el $2005^{13,14}$, en los últimos años han disminuido los niños infectados, especialmente en el grupo de 0 a 9 años ${ }^{5,6}$.

En Chile, los primeros casos de VIH/SIDA en niños fueron detectados en base a sospecha clínica y confirmados por serología ${ }^{3,15}$. El primero, detectado en 1987 fue un niño de 12 años que durante una cirugía por quemaduras eléctricas recibió cinco transfusiones, resultando dos de ellas positivas para $\mathrm{VIH}^{15}$. Con este caso se implementó en 1987 el tamizaje para VIH en todos los bancos de sangre del país. Los primeros casos descritos por transmisión vertical en 1989 fueron un par de gemelos cuya madre adquirió el VIH durante el embarazo por transfusiones ${ }^{15}$.

En 1990 la Sociedad Chilena de Pediatría crea el Comité de VIH/SIDA Pediátrico y sus objetivos han sido la difusión de esta patología, la pesquisa de niños expuestos al VIH y de los infectados con VIH, así como la elaboración de pautas de estudio y manejo, más la prevención y tratamiento de ellos. Para ello este Comité trabaja en conjunto con el Departamento de Programas de ITS y VIH/SIDA (VIH-DIGERA, ex CONASIDA), MINSAL en el Programa Pediátrico de Atención VIH/SIDA, con representantes de todas las regiones del país.

En 1992 comienza la aplicación en los recién nacidos/niños expuestos al VIH (hijos de madres infectadas) de la técnica de Reacción en Cadena de la Polimerasa en la detección del ácido nucleico (AN) del virus (VIH-ADN-RCP) (hasta 1996 en Virología de la Universidad de Chile y desde 1997 a la fecha en el ISP). Como los anticuerpos pasivos persisten hasta los 18 meses, en menores de esa edad la presencia de anticuerpos no permite distinguir si se trata de una inmunidad pasiva (IgG materna) o activa (propios por estar infectado). Por ello en los recién nacidos y niños expuestos al VIH que son detectados en la vida postnatal y que son menores de esa edad, sólo se puede confirmar la infección si se detecta en sangre o tejidos el VIH o sus componentes (AN, antígeno p24) ${ }^{16}$.

En 1995 comienza en Chile la aplicación del PPTV del VIH. En 1994, un estudio en EUA y países europeos 
logró disminuir en 2/3 la transmisión vertical del VIH con la administración del antiretroviral (ARV) Zidovudina (ZDV) durante el embarazo, parto y en el recién nacido (Protocolo PACTG-Pediatric AIDS Clinical Trials Group-076) ${ }^{9}$. Actualmente los PPTV del VIH incluyen, además de los ARVs, varias otras medidas eficaces en la prevención de la trasmisión vertical ${ }^{9-14}$.

En 1996 se inició el uso de Terapia Antiretroviral (TARV) en todos los niños que tuvieran indicación, al comienzo con biterapia [2 Inhibidores de Transcriptasa Reversa Análogos de Nucleosidos (ITRANs)] y desde 1998 con triterapia [2 ITRANs + 1 Inhibidor de Transcriptasa Reversa No Nucleosido (ITRNN) o 1 Inhibidor de Proteasa (IP),] con los ARVs disponibles en ese momento [ZDV, Didanosina (ddI), Lamivudina (3TC), Nevirapina (NVP) y luego Efavirenz (EFV) y Lopinavir/ritonavir (LPV/rtv) $]^{17-19}$. En un comienzo existían pocos ARVs posibles de usar en niños ${ }^{17,18}$. Actualmente existen 19 ARVs que han sido aprobados para menores de 12 años y 6 más aprobados para adolescentes, en quienes, además, se pueden usar algunos ARVs coformulados ${ }^{18,19}$. Actualmente la TAR consiste en una triterapia con un esqueleto de 2 ITRANs más un tercer ARV que puede ser un ITRNN, un IP, un Inh Integrasa (Inh Integr) o un Inhibidor de Entrada ${ }^{18-20}$.

El año 2005 el MINSAL dicta la Norma de Prevención de la Trasmisión Vertical del VIH con cambios el 2009 y $2013^{13,14}$. En Chile, actualmente el PPTV del VIH incluye: a toda embarazada se le debe ofrecer en la primera consulta, en forma obligatoria, el examen de VIH; si el examen resulta negativo, pero existen factores de riesgo, hacer un $2^{\circ}$ examen en la semana 32 34; si resulta infectada ofrecerle TARV preventiva de la transmisión vertical del VIH y/o terapéutica de su infección (actualmente con triterapia en la embarazada y ZDV o ZDV + NVP en el recién nacido), cesárea electiva y que la rotura de membranas no sea mayor de $4 \mathrm{~h}$; en el recién nacido, reemplazar la lactancia materna por leche artificial y seguir con el protocolo de ARVs preventivos. El parto podría ser vaginal sólo si la carga viral $(\mathrm{CV})$ es indetectable $\mathrm{o}<1.000$ copias $/ \mathrm{mL}$ en la semana 34, la TARV se inició en la semana 24, o antes, la edad gestacional es de 37 semanas, el feto es único, en presentación cefálica, y con condiciones obstétricas favorables (Norma del 2013) (13,14. $^{1}$.

\section{Objetivos del Comité de VIH/SIDA Pediátrico}

- Difusión: se efectúa mediante la dictación de cursos, simposios y talleres impartidos en las distintas regiones del país y mediante la edición de material escrito.

- Pesquisa:

- De los recién nacidos: El conocer si una mujer que se embaraza es ya VIH (+) o detectar la infección en ella durante el embarazo permite la implementación de PPTV y evitar en gran parte el nacimiento de nuevos niños infectados.

- En la vida postnatal, la pesquisa puede hacerse por antecedentes familiares de riesgo o de alguien con infección VIH (ej. alguno de los padres).

- Del niño ya infectado con VIH (por trasmisión vertical u horizontal) se puede hacer por antecedentes familiares (factores de riesgo, alguno de los padres o familiar infectado, etc.), antecedentes personales de riesgo (drogadicción, prostitución, transfusiones, etc.) o, más frecuente, por antecedentes clínicos de patologías previas (cuadros respiratorios y/o diarreas frecuentes), o por las manifestaciones clínicas y/o de laboratorio por las que consultan (hepato-esplenomegalia, infección por algún agente oportunista, etc.); menos frecuente, por solicitud del examen de VIH.

- Elaboración de pautas de evaluación, estudio y manejo, usadas en el seguimiento ${ }^{21}$. Se ha trabajado con el MINSAL en la elaboración de Normas de PPTV del VIH de los años 2005, 2009 y 2013, y en las Guías de Tratamiento Antiretroviral de esos mismos años ${ }^{8,13,14}$.

\section{Programa Pediátrico de Atención VIH/SIDA}

Cuando se detecta un recién nacido o niño expuesto al VIH o infectado con VIH se lo debe enviar al Programa Pediátrico del Servicio de Salud o del Hospital Pediátrico correspondiente.

Previo al envío, se les hace una evaluación clínica y de laboratorio con exámenes generales, infectológicos (Citomegalovirus en orina, y serología para VDRL, hepatitis B y C, toxoplasma o Chagas sólo si alguno(s) de esos exámenes estuviera positivo en la madre), inmunológicos (CD4) y virológicos ( $\mathrm{RCP}$, antigenemia y serología), con toma en las 48-72 h de vida de la primera muestra de sangre para VIH-ADN-RCP ${ }^{8,13,14}$. En el manejo del recién nacido, por la posibilidad de transmisión del virus por la leche, hay que suspender la lactancia materna y por nodrizas [en Chile el MINSAL, aporta fórmulas de inicio durante los primeros 6 meses de vida a los hijos de madre VIH $(+)$ usuarios del sistema]; la vacuna BCG sólo se coloca si los valores de CD4 o el número de leucocitos son normales para la edad, o si el estudio virológico específico descarta la infección por VIH. Además, se inicia la TARV Preventiva de la trasmisión vertical del VIH (sólo con ZDV o, además, con NVP) (12-14). El caso debe ser notificado 
al MINSAL y comunicado al Programa Pediátrico al que será derivado.

- En el Programa Pediátrico, a los recién nacidos expuestos al VIH se los sigue hasta que se confirma o descarta la infección mediante la técnica de VIHADN-RCP (tablas 1 y 2). Si se confirma la infección siguen en control hasta los 18 años, edad en que pasan a control en el Programa de Adultos. Si se descarta la infección por VIH pasan a control de niño normal, pero el descarte definitivo se hace con un examen serológico de VIH a los 18 meses de edad ${ }^{8,16}$.

- En el Programa Pediátrico, a los recién nacidos con infección confirmada y a los niños infectados detectados en la vida postnatal, se les sigue con evaluaciones periódicas clínicas (general y por especialistas) y de laboratorio [exámenes generales, infectológicos, inmunológicos (cuantificación de inmunoglobulinas y CD4) y virológicos $(\mathrm{CV})]^{8}$.

La evaluación clínica e inmunológica (CD4) inicial permite la etapificación de los niños infectados con VIH según la Clasificación CDC $1994^{7}$.

La evaluación clínica, inmunológica y virológica (CD4 y CV cada 3-4 meses) permite estimar el pronóstico, adecuar el manejo preventivo y terapéutico, decidir el inicio de TARV, seguir la respuesta a ella y efectuar los cambios, si necesitara ${ }^{8,18-20}$.

El manejo es multidisciplinario y comprende medidas preventivas de infecciones oportunistas como Pneumocystis jirovecii e indicación de vacunas, y medidas terapéuticas de la infección y complicaciones ${ }^{22-26}$. La terapia de la infección VIH se inicia de acuerdo a la Guía Clínica de Síndrome de Inmunodeficiencia Adquirida, VIH/SIDA, MINSAL (guías 2005, 2009, 2013) ${ }^{8}$.

Según todas las guías de TARV, los niños que están en etapa clínica de SIDA y/o en etapa inmunológica 3 (inmunosupresión severa) deben recibir TARV, independiente de la carga viral $\left.{ }^{8,18-20}\right)$. La indicación de TARV en niños que están en otras etapas clínicas y/o inmunológicas ha variado con el tiempo: desde un inicio más tardío a uno más precoz. Según la Guía del MINSAL 2013, se debe iniciar TARV en todo niño infectado con VIH que sea menor de 1 año, independiente de la clínica, CD4 o la carga viral; en los niños mayores de un año la decisión de inicio de terapia se basa en la clínica y/o en los exámenes de CD4. Para el cambio de terapia, si no es por efecto adverso, se basa, además, en la carga viral ${ }^{8,18-20}$.

Se debe evaluar la adherencia y cumplimiento de la TARV, la tolerancia, y los efectos adversos e interacciones con drogas.

\section{Resultados: Cohortes VIH/SIDA en niños y adolescentes}

El Comité de VIH/SIDA Pediátrico ha hecho dos evaluaciones de los niños/adolescentes que controla: la primera Cohorte contempló desde el año 1987 al 2008 y la segunda desde 1987 a Agosto 2014 27-28.

Según la Cohorte 2, de los 375 niños detectados hasta el 2014, 245 niños y adolescentes se mantenían en control, 18 adolescentes pasaron al Programa de Adultos, 73 habían fallecido por causas relacionadas al VIH/SIDA y por otras causas, y 39 se habían perdido de seguimiento ${ }^{28}$.

La aplicación progresiva desde 1995 de PPTV del VIH en binomios madre-hijo se tradujo en una reducción significativa de la trasmisión vertical desde el $30-35 \%$ anterior a esa fecha, sin protocolos, a un $2 \%$

Tabla 1. Muestras para diagnóstico específico de VIH en recién nacidos expuestos, hijos de madre VIH (+)

$1^{\text {a }}$ muestra para VIH-ADN-RCP: antes de las $48-72 \mathrm{~h}$ del nacimiento (sangre periférica, no del cordón).

$2^{\text {a }}$ muestra para VIH-ADN-RCP: a las 2-4 semanas de vida (o lo antes posible frente a un resultado positivo de la primera muestra).

$3^{\mathrm{a}}$ muestra para VIH-ADN-RCP: tomar lo antes posible si la primera y/o la segunda muestra resultan positivas, y no más tarde de los 3 a 4 meses de edad si las dos primeras resultan negativas

Esto es válido siempre que el RN Expuesto no reciba alimentación a pecho materno o por nodrizas.

El estudio virológico para diagnóstico de la infección por VIH se efectúa en el Instituto de Salud Pública (ISP).

Tabla 2. Infección por VIH en Niños: Diagnóstico de Laboratorio (ISP; Diciembre 1997)

\begin{tabular}{lll}
\hline Edad & Confirmación Infección & Descarte Infección \\
\hline Recién nacidos & Al menos 2 muestras con RCP $(+)$ sin considerar & Al menos 2 muestras con RCP (-) sin considerar \\
& $1^{\text {a } \text { muestra de antes de las 48 h de vida }}$ & $1^{\text {a } \text { muestra de antes de las 48 h de vida }}$ \\
Niños $\geq 1$ mes $y<18$ meses & 2 RCP deben ser $(+)$ & 2 RCP deben ser (-) \\
Niños $\geq 18$ meses & Basta 1 RCP $(+)$ y/o serología VIH $(+)$ confirmada & Basta 1 RCP (-) y/o serología VIH (-) \\
\hline
\end{tabular}

*Siempre que no estén con alimentación a pecho por la madre o por nodrizas. El estudio virológico para diagnóstico de la infección por VIH se efectúa en el Instituto de Salud Pública (ISP). 
entre el año 1998 al 2005, con protocolos, cifra que se mantiene hasta ahora en los binomios en que se ha aplicado (en la embarazada primero sólo con ZDV, luego con biterapia, ZDV + 3TC, y desde 2005 con triterapia siendo los esquemas que más se han usado los con ZDV + 3TC como esqueleto y NVP o LPV/rtv como tercera droga; actualmente se han agregado otros ARVs en la embarazada $)^{9,12-14}$. Entre los años 1998 al 2005 nacieron sólo 8 niños infectados de 401 binomios madre-hijo en PPTV, lo que contrasta con los 162 niños VIH (+) que fueron detectados hasta fines del 2013 y que nacieron en ese período del estudio de madres $\sin \mathrm{PPTV}^{30}$.

La puesta en vigencia el año 2005 de la Norma de Prevención de la Trasmisión Vertical del VIH significó una mayor disminución de nuevos niños infectados con VIH. Según la Cohorte 2, el 80\% de los niños infectados fue detectado antes del 2005, la mayoría en la vida postnatal principalmente por patología o alteraciones de laboratorio del niño, y el 20\% entre el 2006 y $2014^{13-15,27,28}$. El 20\% detectado entre el 2006 y el 2014 se explica por niños nacidos antes del 2005 y que por ser de lento progreso ${ }^{31}$ se pesquisan en la vida postnatal por clínica y, además, por niños nacidos entre el 2005 y 2014, detectados por diagnóstico tardío del VIH en la madre, durante el parto o en el período postparto, o detectados en la vida postnatal por patología del niño; un pequeño porcentaje fue diagnosticado por ingreso a PPTV ${ }^{28,30}$. Es así como entre el año 2006 y hasta Julio del 2014 se han detectado 127 niños VIH (+) y de los 63 que nacieron en esos años, 59 se infectaron por transmisión vertical; de ellos sólo la mitad ${ }^{30}$ recibió PPTV, y sólo en $1 / 3$ de los casos fue completo. De las embarazadas sin PPTV, la mitad ${ }^{14}$ no lo recibió porque tuvieron un examen VIH (-) durante la gestación $y$ en otros casos hubo fallas en el sistema de salud como

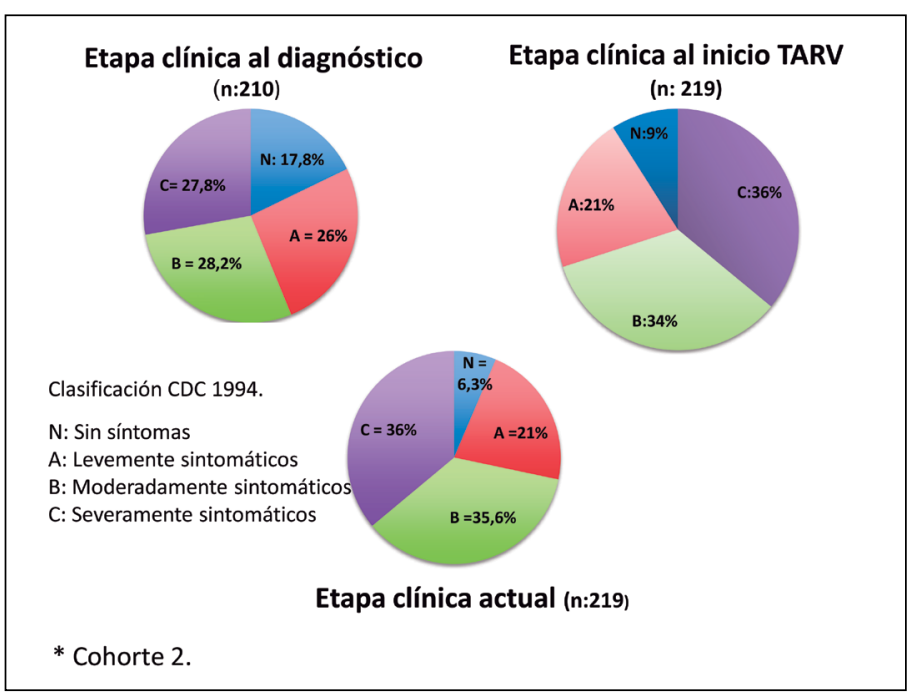

Figura 1. Evolución etapas clínicas, Clasificación CDC 1994* no ofrecimiento del test, no rescate del resultado, no ofrecimiento de la TARV preventiva. Todo esto ha significado que se mantenga la transmisión en $\pm 2 \%$ en aquellos binomios con prevención de la transmisión vertical completa o incompleta ${ }^{30}$.

La mayoría de los niños infectados con VIH ha sido detectado por clínica (47\% hasta el año 2008, $42 \%$ hasta el año 2014) $)^{27,28}$. Según la Cohorte del 2014 la detección en base a antecedentes maternos se hizo en un $35 \%$ y por ingreso a PPTV en un $10 \%{ }^{28}$.

Hasta el año 2014, de 245 niños infectados con VIH por transmisión vertical, el $46 \%$ fue diagnosticado el año de nacimiento; los demás lo fueron en edades más tardías generalmente por manifestaciones clínicas. Del $80 \%$ de niños detectados antes del 2005 sólo un tercio de ellos fue diagnosticado el año de nacimiento. Del 20\% de niños pesquisados desde el año 2006 al 2014, el $50 \%$ lo fue en el año de nacimiento ${ }^{28}$.

El año 2014, el promedio de edad de los pacientes en control era de 11 años (rango de 1 a 21 años): el 30\% menores de 10 años y el 70\% mayores de esa edad, es decir las dos terceras partes de los pacientes contagiados verticalmente estaban ya en la adolescencia ${ }^{28}$.

La mayoría de los niños detectados por clínica lo fueron por manifestaciones inespecíficas (respiratorias, digestivas e infecciones) $)^{32-34}$. Entre las infecciones, las más frecuentes fueron las bacterianas (neumonías, septicemias, meningitis), por agentes habituales de la infancia, $y$ las por agentes oportunistas, principalmente por Citomégalovirus y cándidas, menos frecuentes, por Pneumocystis jirovecii, Cryptosporidium, Virus Herpes Simple, Mycobacterium tuberculoso y Mycobac-

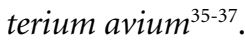

Hasta el 2008, el 41\% de los niños había sido detectado en etapa de SIDA y el 59\% en etapas No SIDA. El 2014 estas cifras fueron de $27,8 \%$ y $72 \%$ respectivamente [etapa $\mathrm{N}(17,8 \%)$, etapa $\mathrm{A}(26 \%)$ y etapa $\mathrm{B}$ $(28,2 \%)]$ (Clasificación CDC 1994) $8,27,28$.

Hasta el 2014 (Cohorte 2), el 92\% de los niños y adolescentes se encontraban en TARV y el $8 \%$ no cumplía con los criterios de inicio. De los pacientes en TARV, la edad promedio de inicio fue 3 años y 2 meses (rango: 1 mes a 15 años). Hasta esa fecha las tres cuartas partes de los pacientes había usado 1 o 2 esquemas de TARV, con esquemas, en general, de primera línea. La minoría había necesitado esquemas de tercera línea o de rescate ${ }^{28}$. Más del $75 \%$ tuvo adherencia excelente o buena, en el $8 \%$ fue mala, y en el resto, regular ${ }^{27}$.

Las etapas clínicas (Clasificación CDC 1994) al diagnóstico, al inicio de la TARV y en el momento de la evaluación (año 2014) se muestran en la figura 1. De los pacientes detectados en etapas NO SIDA ( $44 \%$ en etapas $\mathrm{N}$ y A y $28 \%$ en etapa B) destacan las infecciones respiratorias, altas y bajas, frecuentes. De los pacientes detectados en etapa C, SIDA, (28\%) las infecciones más 
frecuentes fueron neumonía y enfermedad diseminada por citomegalovirus, neumonía por Pneumocystis jirovecii, neumonías bacterianas a repetición y septicemias severas. Al inicio de la TARV las etapas B y C habían aumentado a $34 \%$ y $36 \%$ respectivamente ${ }^{28}$.

Las etapas inmunológicas (Clasificación CDC 1994) al diagnóstico, al inicio de la TARV y en el momento de la evaluación (año 2014), se muestran en la figura 2. Se observa que al momento del diagnóstico, el porcentaje de niños que se encontraba en cada una de las etapas inmunológicas era más o menos similar $(30 \%$ o más). Al inicio de la TARV el porcentaje de niños con compromiso inmunológico severo (etapa 3 ) había aumentado a $67 \%$, por disminución de las etapas $2(22 \%)$ y especialmente de la $1(11 \%)$. Con el uso de la TARV, y la consiguiente recuperación inmune, aumentaron a $70 \%$ los pacientes en etapa 1 (sin supresión inmune) y disminuyeron a $8 \%$ los niños en etapa 3 . No hubo cambios en el porcentaje de la etapa inmunológica $2^{28}$.

Con el uso de TARV en los niños se ha logrado disminuir las infecciones, especialmente las oportunistas primarias, ha mejorado la calidad de vida, $y$ con la prolongación de ella han aparecido las infecciones oportunistas por agentes que quedan latentes y los cánceres $^{36,38}$.

De los 375 pacientes pediátricos diagnosticados por el ISP desde 1987 al año 2014, han fallecido 73 (19,6\%), por causas relacionadas al VIH y por otras causas. En el primer período (antes año 2000) fallecieron treinta $y$ tres casos (45\%) por causas principalmente infecciosas $(70 \%)$, especialmente por agentes oportunistas y menos por bacterias; en el segundo período (2000 al 2005), fallecieron 25 casos (34\%), observándose una disminución de las infecciones como causa de muerte, igualándose las oportunistas y las bacterianas, y aparecen los cánceres; en el tercer período (2005 al 2014), fallecieron 15 niños (20,5\%), con una mayor disminución de las infecciones, especialmente de las oportunistas, y aumento de los cánceres como causa de muerte (figura 3). En el primer periodo el $100 \%$ de los fallecidos no recibió TARV, en el segundo sólo un $36 \%$ la usó, y en el tercero un $87 \%$ recibió TARV (figura 3$)^{28,38,39}$.

\section{Discusión}

En Chile, el trabajo conjunto del Comité de VIH/ SIDA, SOCHIPE, con el MINSAL en el Programa de Atención VIH/SIDA ha logrado varios progresos en el control de los niños y adolescentes infectados con VIH.

Uno ha sido la aplicación progresiva a partir de 1992 de la técnica VIH-ADN-RCP como herramienta diagnóstica del VIH en lactantes, mucho antes que otros países latinoamericanos. Con la aplicación de esta técnica molecular disminuyó la edad de confirmación o descarte de la infección por VIH, desde 26-27 meses de edad en 1989 a 3-4 meses en la actualidad ${ }^{6,16}$. El que se tome la primera muestra antes de las 48$72 \mathrm{~h}$ de vida (en otras guías es a partir de los 15 días de edad ${ }^{18}$ ) permite determinar el momento en que se produce la infección: in útero, factor de mal pronóstico para la evolución del niño, o tardíamente en el embarazo o periparto, de mejor pronóstico. Sin embargo, el mayor uso de la TARV preventiva de la transmisión vertical puede hacer que la primera muestra neonatal pueda resultar negativa ${ }^{40}$.

El PPTV se comenzó a aplicar en 1995, 10 años antes de la Norma, bajando la trasmisión a un $2 \%$ en los binomios en protocolo, lo que demuestra la efectividad del PPTV ${ }^{29,30}$.

La Norma de Prevención de la Transmisión Vertical del VIH del $2005^{13,14}$ continúa la disminución: sólo el 20\% de los detectados se encuentra entre el 2006

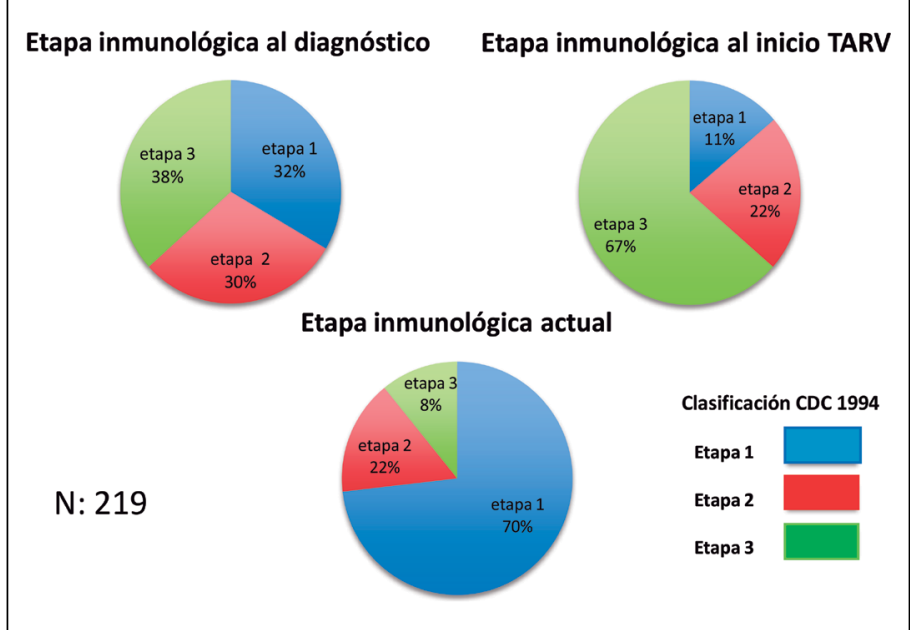

Figura 2. Evolución etapas inmunológicas, Clasificación CDC 1994*.

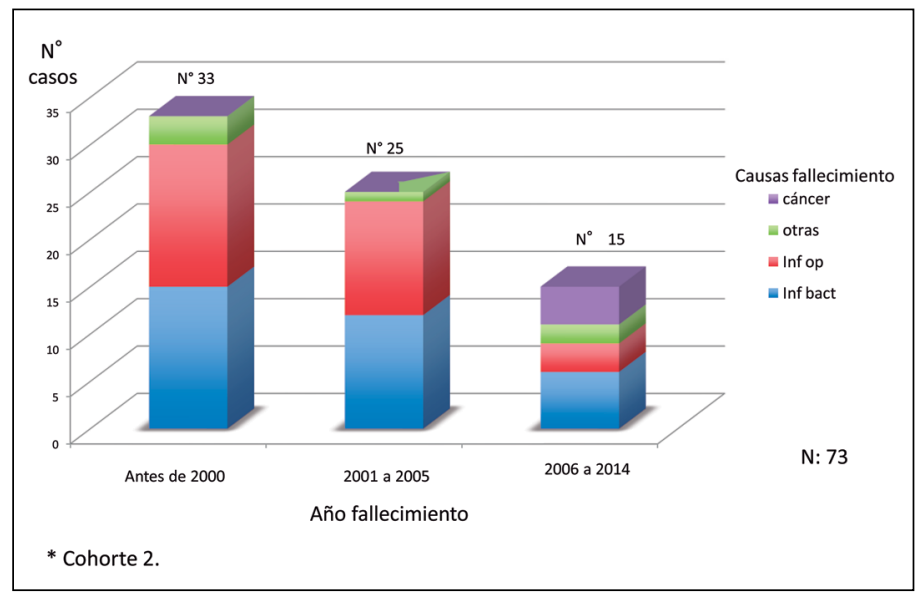

Figura 3. $\mathrm{N}^{\circ}$ de fallecidos y causas de muertes por VIH/SIDA, por períodos* 
Tabla 3. Evolución trimodal infección por VIH en niños

\begin{tabular}{lccc}
\hline Evolución & Rápida & Intermedia & Lenta \\
\hline Frecuencia & $10-15 \%$ & $50-70 \%$ & $10-15 \%$ \\
Deficiencia Inmune & Precoz (pocos meses) & Tardía (meses, pocos años) & Más tardía (años) \\
Deterioro clínico & $<2$ años edad & 5 años edad & 8 años edad \\
Manifestaciones Iniciales & $\begin{array}{l}\text { Antes 1 año: Infs. oportunistas, } \\
\text { encefalopatía precoz, falla en } \\
\text { prosperar, diarrea }\end{array}$ & $\begin{array}{l}\text { Antes } 5 \text { años: manifestaciones } \\
\text { leves o infecciones bacterianas, } \\
\text { neumonitis intersticial linfoide, } \\
\text { parotiditis }\end{array}$ & $\begin{array}{c}\text { Antes } 8 \text { años: ausentes o pue- } \\
\text { den no progresar }\end{array}$ \\
Desarrollo de SIDA & En meses, $<1$ a 2 años & En años & En muchos años \\
Sobreviva a los 5 años (sin TARV) & $50 \%$ & $70-75 \%$ & $>75 \%$
\end{tabular}

y 2014, explicable, en parte, por diagnóstico tardío y terapia incompleta o por haber tenido un solo test de VIH que resultó negativo ${ }^{28}$.

La divulgación del tema a lo largo del país permitió una mejoría en la pesquisa con disminución de los niños detectados en etapa de SIDA de $41 \%$ el año 2008 a 28\% el 2014, y aumento de los detectados el año de nacimiento de 33\% antes del 2005 a 50\% desde 2006 al $2014^{27,28}$. Muchos de los niños pesquisados después del año de su nacimiento, lo han sido por ser progresores lentos que presentaron manifestaciones tardíamente $(\text { tabla } 3)^{31}$.

En 1998 se cambió la TARV de biterapia a triterapia, mucho antes que los adultos ${ }^{17,18}$. Gracias a la adherencia lograda y a los pocos efectos adversos presentados, hasta el año 2014 la gran mayoría de los niños/ adolescentes había usado sólo 1 o 2 esquemas de TARV y con ARVs de $1^{\mathrm{a}}$ línea ${ }^{28}$.

La efectividad de la TARV se evidenció en una mejoría clínica, inmunológica (aumento de niños en etapa 1 de $11 \%$ a $70 \%$ y disminución de niños en etapa 3 , de $67 \%$ a $8 \%$ ), y virológica (carga viral indetectable antes de los 6 meses de TARV), en una disminución de las infecciones (especialmente de las oportunistas) y de las muertes. Se logró una prolongación de la vida; el año 2014 el 70\% de los niños detectados eran adolescentes $^{28}$.

Hasta el año 2014 cinco de las primeras niñas detectadas se habían embarazado y sus hijos nacieron sin la infección del VIH con el PPTV ${ }^{39,41}$.

A pesar de estos progresos, aún hay desafíos que resolver. Uno de ellos es lograr la eliminación de la transmisión vertical del VIH, meta establecida el año 2010 por la OMS y Naciones Unidas (NU) para las Regiones de las Américas. Entre el 2010 y el 2015 la tasa de transmisión vertical en América Latina y el Caribe disminuyó de $15 \%$ a $8 \%$; 22 países habían alcanzado la meta de eliminación el 2015, siendo Cuba el primero en lograrlo ${ }^{42}$.

En Chile la transmisión disminuyó desde 7,2\% el año 2009 (11 de 152) a 2,5\% el año 2014 (4 de 157) con una tasa de $0,2 / 1000$ nacidos vivos ${ }^{43}$. La cifra acerca a Chile a la meta de eliminación establecida por la OMS y NU ${ }^{42-44}$.

Dificultan lograr esta meta el no cumplimiento total del PPTV, el control tardío o la falta de control del embarazo, un resultado negativo del único test de VIH efectuado durante el embarazo, el uso de ARVs cada vez más diversos en la embarazada ${ }^{12}$ con nacimiento de niños infectados ya con multi-resistencia a algunos de ellos, y el problema de los inmigrantes, especialmente haitianos, por el idioma y porque suelen presentar otras infecciones concomitantes con el VIH, como la hepatitis $\mathrm{B}^{44,45}$.

Contribuirá a lograr la meta un segundo test de VIH en el tercer trimestre del embarazo, a incorporar en las nuevas Normas y Guías del MINSAL.

\section{Conflicto de intereses}

La autora declara no tener conflicto de interés.

\section{Referencias \\ 1.- ONUSIDA. Ultimas estadísticas sobre el estado de la epidemia de SIDA, Julio 2017. \\ 2.- ONUMUJERES. Hechos y cifras, Junio 2016. \\ 3.- Departamento de Epidemiología, División}

de Planificación Sanitaria Ministerio de Salud de Chile. Informe Nacional: evolución de la infección por VIH/SIDA Chile 1984-2012. Rev Chil Infectol 2015, 32 S1:18-43.

4.- Instituto de Salud Pública: Resultados confirmación de infección por VIH, Chile,
2009-2011. Boletín Vol. 2, No. 6, Mayo 2012.

5.- Instituto de Salud Pública. Resultados confirmación de infección por VIH en Chile, 2010-2015. Boletín Vol. 6, No. 11, Noviembre 2016.

6.- Instituto de Salud Pública (ISP), 
Ministerio de Salud, Chile. Informativo trimestral resultados diagnóstico virológico infección VIH pediátrica. Boletines ISPCH: resultados confirmación Infección VIH en Chile.

7.- CDC. Revised Classification system for human immunodeficiency virus infection in children less than 13 years of age. MMWR Morbid Mortal Wkly Rep 1994; 43(RR-12):1-10

8.- $\quad$ Ministerio de Salud. Serie de Guías Clínicas Síndrome de Inmunodeficiencia Adquirida, VIH / SIDA, 1 ${ }^{\text {a }}$ Ed., 2005; 2a Ed., 2009; 3a Ed., 2013.

9.- CDC. Recommendations of the US Public Health Service Task Force on the use of zidovudine to reduce perinatal transmission of human immunodeficiency virus. MMWR Morbid Mortal Wkly Rep 1994;43 (RR-11):1-20,

10.- Bulteryss M, Fowler MG. Prevention of HIV infection in children. Pediatr Clin North Am 2000;47:241-60.

11.- Hudson C N. Elective Caesarean-Section for prevention of vertical transmission of HIV-1 infection. Lancet 1999;353:1030-1.

12.- Recommendations for Use of Antiretroviral Drugs in Pregnant HIV-1Infected Women for Maternal Health and Interventions to Reduce Perinatal HIV Transmission in the United States. Ultima puesta al día, Noviembre 14;2017:1-318.

13.- CONASIDA, Ministerio de Salud, Chile Norma para Prevención de la Transmisión Vertical del VIH, Agosto 2005, 2009 y 2013.

14.- Programa Nacional de Prevención y Control de la infección por VIH/ SIDA e ITS, División de Prevención y Control de Enfermedades, Subsecretaría de Salud Pública, Ministerio de Salud. Norma Conjunta de Prevención de la Transmisión Vertical del VIH y la Sífilis. Rev Chil Infectol 2013;30:259-302.

15.- Wu E. Más de 25 años en Chile de la Infección VIH en Niños y Adolescentes. Rev Chilena Infectol 2015;32(S1):44-55.

16.- Ministerio de Salud, Subsecretaría de Salud Pública, Subsecretaría de Redes Asistenciales. Manual de procedimientos para la detección y diagnóstico de la infección por VIH 2009, pags. 1-223.

17.- Pizzo PhA, Wilfert MC. Antiretroviral Treatment for Children with HIV Infection. In Pediatrics AIDS: The challenge of HIV in infants, children and adolescents. Eds, 2nd Ed., Wilkins and Wilkins, Baltimore, USA 1994:651-87.

18.- AIDS Info: Guidelines for the Use of Antiretroviral Agents in Pediatric HIV Infection. Ultima puesta al día, Noviembre 14, 2017, pags 1-355.

19.- AIDS Info. Guidelines for the Use of Antiretroviral Agents in HIV-1-Infected Adults and Adolescents.Ultima versión
Octubre 2017, pags. 1-298.

20.- PENTA Steering Committe. 1) PENTA 2009 Guidelines for the use of antiretroviral therapy in paediatric HIV-1 infection, HIV Med 2009;10:591-613; 2) Guidelines for treatment of paediatric HIV-1 infection 2015: optimizing health in preparation for adult life. HIV Medicine 2015, 15: 1-42; and 3) Paediatric European Network for Treatment of AIDS Treatment Guideline 2016 update: antiretroviral therapy recommended for all children living with HIV, HIV Med 2016;133-4.

21.- Wu E. Infección por el virus de la inmunodeficiencia humana en el binomio madre-hijo, Guía del Comité VIH/SIDA Pediátrico, SOCHIPE; 2000, 1-158 más anexos.

22.- Laufer M, Scott G B. Medical management of HIV diseases in children. Pediatr Clin North Am 2000;47:127-52.

23.- Wu E. Infección por VIH en Niños. En libro SIDA, 5a Ed, Sepúlveda C y Afani A Eds., Editorial Mediterráneo, Santiago, Chile, 2017:234-57.

24.- Wu E. Vacunas en Personas con Condiciones Especiales de Salud. Apuntes de Práctica Clínica en Pediatría. Alarcón T y Vargas L Eds, 9ª Ed, Dpto. de Pediatría y Cirugía Infantil Occidente, Facultad de Medicina, U. Chile y Servicio de Pediatría Hospital San Juan de Dios. 2013;454-70.

25.- AIDS Info. Guidelines for the Prevention and Treatment of Opportunistic Infections Among HIV-Exposed and HIV-Infected Children. Ultima puesta al día Diciembre 2016;1-390.

26.- AIDS Info. Guidelines for Prevention and Treatment of Opportunistic Infections in HIV-Infected Adults and Adolescents. Ultima puesta al día Agosto 2017;1-428.

27.- Larrañaga $C, \mathrm{Wu} E$, Alvarez $\mathrm{AM}$, Chavez A, Vizueta E, PeñaAm, Galaz MI, Bravo CL, por Comité VIH/SIDA Pediátrico, Chile. "Evaluación de la Cohorte Nacional de VIH / SIDA Pediátrico, Chile 1987-2008". Presentado en XIII Congreso Latinoamericano de Infectología Pediátrica, SLIPE, IV Congreso Ecuatoriano de infectología, Guayaquil, Ecuador, 12-15/08/09. Libro de resúmenes, VIH-5:35, y en XII Taller de VIH / SIDA Pediátrico, Santiago, Chile, 28-29/05/2010.

28.- González M, Galaz MI, Chavez A, et al, por Comité VIH/SIDA Pediátrico, SOCHIPE. Cohorte Pediátrica VIH. XXXI Congreso Chileno de Infectología, Puerto Varas, 12-15/XI/2014.

29.- Chávez A, Álvarez AM, Wu E, Peña A, Vizueta E, y Comité Nacional de VIH / SIDA Pediátrico, Sociedad Chilena de Pediatría. Evolución de la Transmisión Vertical de la Infección por VIH en Chile. Rev Chil Infectol 2007; 24:368-371.
30.- Chavez A por Comité de VIH/SIDA Pediátrico, SOCHIPE. Transmisión Vertical del VIH en Chile. Presentado en Curso en XVII Curso Nacional de VIH, Santiago, 29 y 30 de Agosto 2014.

31.- Morris CR, Araba-Owoyele L, Spector SA, et al. Disease patterns and survival after acquired immunodeficiency syndrome diagnosis in human immunodeficiency virus-infected children. Pediatr Infect Dis J 1996;15:321-8.

32.- Tassara R, Alarcón $T$, Larrañaga $C$, Wu E, Alvarez AM. Patología digestiva en niños infectados con el virus de inmunodeficiencia humana (VIH), en Santiago de Chile. Rev Med Chile 2003;131:19-24.

33.- Brockman P, Viviani T, Peña A. Compromiso pulmonar en la infección por virus inmunodeficiencia humana en niños. Rev Chil Infect 2007;24:301-5.

34.- Wu E. Compromiso Pulmonar por VIH. En Alergia e Inmunología Respiratoria. Quezada A y Zenteno D. Eds., $1^{\text {a }}$ Ed. Editorial Mediterráneo 2013;229-37.

35.- Peña $A M$, Larrañaga $C$, Luchsinger $F$, Villarroel J, Chavez A, Wu E, y Comité Nacional de SIDA Pediátrico, Sociedad Chilena de Pediatría. Enfermedad por Citomegalovirus en Niños Chilenos Infectados con el Virus de la Inmunodeficiencia Humana. Rev Chil Infectol 2007;24: 477-84.

36.- Villarroel J, Vizueta E, Álvarez AM, et al. Tuberculosis en SIDA y Pediatría. Rev Chil Infect 2007;24:472-6.

37.- Peña AM, Larrañaga $C, W u$ E, Álvarez AM, et al, por Comité VIH/SIDA Pediátrico, Chile. "Eventos Oportunistas y Enfermedad por Citomegalovirus en Niños Chilenos Infectados con VIH". XIII Congreso Latinoamericano de Infectología Pediátrica, SLIPE, IV Congreso Ecuatoriano de Infectología, Guayaquil, Ecuador, 12.15/08/09. Libro de resúmenes, VIH-5, pag 37. Presentado en XII Taller de VIH / SIDA Pediátrico, Santiago, Chile, 28-29/05/2010.

38.- Villarroel J, Álvarez AM, Chávez A, Cofré $\mathrm{J}$, et al. Incidencia de cánceres en niños chilenos infectados con VIH. Rev Chil Infectol 2015;32:672-6.

39- Wu E, Galaz MI, Larrañaga C, Chávez A, et al en representación Comité VIH/SIDA Pediátrico, SOCHIPE. Infección por VIH/ SIDA en niños y adolescentes: Cohorte chilena 1987-2014. Rev Chil Infectol 2016;33(S1):11-9.

40.- Puthankit T, Rojanwiwat T. Delayed HIV DNA PCR detection among infants received combination ART prophylaxis. Conference on Retroviruses and Opportunistic Infections 2017.

41.- Villarroel J, Álvarez AM, Salvador F, Chvez A, Wu E, Contardo V. Mujeres jóvenes con infección por VIH adquirida 
por transmisión vertical. Espectativas de tener hijos no infectados. Rev Chil Infectol 2016;33:650-5.

42.- Programa Conjunto de las Naciones Unidas sobre el VIH/SIDA y Organización Mundial de la Salud. Informe mundial de avances en la lucha contra el SIDA 2016.

43.- United Nations Department of Chile.

Chile País 20102011201320142015.

Porcentaje notificado por el país, 2015.

Prevalencia estimada de la infección por el

VIH (mujeres de 15-49 años), 2015.
44.- La Tercera Comunicado de Prensa 2015. Chile a un paso de eliminar la transmisión de VIH entre madre e hijo 20/09/2015.

45.- Harding A. U.S. Perinatal HIV Infection Fell to 69 Infants in 2013: CDC. JAMA Pediatr 2017.Mar 22. 